\title{
Validation of modified World Health Organization classification for pregnant women with heart disease in a tertiary care center in southern Thailand
}

Chitkasaem Suwanrath'

Putthaporn Thongphanang'

Sutham Pinjaroen'

Saranyou Suwanugsorn ${ }^{2}$

'Department of Obstetrics and Gynecology, ${ }^{2}$ Department of Internal Medicine, Faculty of Medicine, Prince of Songkla University, Hat Yai, Songkhla, Thailand
This article was published in the following Dove Press journal: International Journal of Women's Health

Purpose: To validate the modified World Health Organization (WHO) classification in pregnant women with congenital and acquired heart diseases.

Patients and methods: The database of pregnant women with heart disease, who delivered at Songklanagarind Hospital between January 1995 and December 2016, was retrieved from the Statistical Unit, Department of Obstetrics and Gynecology, along with the Hospital Information System of Songklanagarind Hospital, Faculty of Medicine, Prince of Songkla University. Each patient was retrospectively classified according to the modified WHO classification of maternal cardiovascular risk. Comparison of maternal and fetal outcomes among the modified WHO classes were analyzed using the chi-square test or Fisher's exact test and one-way ANOVA test. A $p$-value of $<0.05$ was considered statistically significant.

Results: A total of 331 cases were studied: 157 cases with congenital heart disease and 174 cases with acquired heart disease. There were 48, 173, 32 and 78 cases in the modified WHO class I, II, III and IV, respectively. Congestive heart failure was the most common complication. The overall maternal mortality rate was $3.6 \%$, all of which were in the modified WHO class IV. Maternal cardiovascular events occurred in $24.2 \%$ of cases, increasing rates with higher modified WHO class: $4.2 \%, 15.0 \%, 25.0 \%$ and $56.4 \%$ in class I, II, III and IV, respectively $(p<0.001)$. Adverse fetal outcomes including preterm delivery, low birth weight, small for gestational age and neonatal intensive care unit admission were also significantly increased in class III and IV $(p<0.05)$.

Conclusion: The modified WHO classification is useful not only for obtaining a cardiovascular risk assessment in pregnant women with heart disease but also for predicting adverse fetal outcomes. It must, therefore, be implemented into routine care service at all levels of health care systems.

Keywords: heart disease, cardiovascular complications, pregnancy, heart failure, maternal mortality

\section{Introduction}

Heart disease is a leading cause of maternal mortality in developed countries as well as some developing countries, including Thailand, with incidence varying from $1 \%$ to $4 \% .{ }^{1}$ Pregnancy induces hemodynamic changes to meet the demands of the mother along with the fetus, which include increases in blood volume and cardiac output and reduction in systemic vascular resistance coupled with blood pressure. ${ }^{1}$ This can then lead to an increase in maternal and perinatal morbidity and mortality.
Department of Obstetrics and

Gynecology, Faculty of Medicine, Prince of Songkla University, Hat Yai, Songkhla

90110 , Thailand

Tel +667445 I 201

Fax +6674429617

Emailschitkas@yahoo.co.uk
International Journal of Women's Health 2018:10 47-53

(c) (7) (5) 2018 Suwanrath et al. This work is published and licensed by Dove Medical Press Limited. The full terms of this license are available at https://www.dovepress.com/terms.php cc. hereby accept the Terms. Non-commercial uses of the work are permitted without any further permission from Dove Medical Press Limited, provided the work is properly attributed. For permission hereby accept the Terms. Non-commercial uses of the work are permitted without any further permission from Dove Mercial use of this work, please see paragraphs 4.2 and 5 of our Terms (https://www.doveperss.com/terms.php).
for commercial 
In 2011, the European Society of Cardiology guidelines on the management of cardiovascular diseases during pregnancy recommended to estimate maternal risk according to the modified World Health Organization (WHO) classification. ${ }^{2}$ This risk classification integrates all known maternal cardiovascular risk factors including underlying heart disease and comorbidity. ${ }^{2}$ The patients are classified as very low risk (class I), low to moderate risk (class II), high risk (class III) and extremely high risk (class IV), in which pregnancy is contraindicated. ${ }^{2}$ WHO class I consists of 1) uncomplicated, small or mild pulmonary stenosis, patent ductus arteriosus and mitral valve prolapse; 2) successfully repaired simple lesions (atrial or ventricular septal defect, patent ductus arteriosus, anomalous pulmonary venous drainage); and 3) atrial or ventricular ectopic beats, isolated. WHO class II (if otherwise well and uncomplicated) includes 1) unoperated atrial or ventricular septal defect; 2) repaired tetralogy of Fallot; and 3) most arrhythmias. WHO class II-III (depending on individual) consists of 1) mild left ventricular impairment; 2) hypertrophic cardiomyopathy; 3 ) native or tissue valvular heart disease not considered WHO I or IV; 4) Marfan syndrome without aortic dilatation; 5) aorta $<45 \mathrm{~mm}$ in aortic disease associated with bicuspid aortic valve; and 6) repaired coarctation. WHO class III includes 1) mechanical valve; 2) systemic right ventricle; 3) Fontan circulation; 4) cyanotic heart disease (unrepaired); 5) other complex congenital heart disease; 6) aortic dilatation $40-45 \mathrm{~mm}$ in Marfan syndrome; and 7) aortic dilatation $45-50 \mathrm{~mm}$ in aortic disease associated with bicuspid aortic valve. Finally, for WHO class IV (pregnancy contraindicated), there are seven conditions including 1) pulmonary arterial hypertension of any cause; 2) severe systemic ventricular dysfunction (left ventricular ejection fraction $<30 \%$, New York Heart Association III-IV); 3) previous peripartum cardiomyopathy with any residual impairment of left ventricular function; 4) severe mitral stenosis and severe symptomatic aortic stenosis; 5) Marfan syndrome with aorta dilated $>45 \mathrm{~mm}$; 6) aortic dilatation $>50 \mathrm{~mm}$ in aortic disease associated with bicuspid aortic valve; and 7) native severe coarctation. ${ }^{2}$ Since then, validation of the guidelines has been performed, which has mainly focused on pregnant women with congenital heart disease, compared with other risk assessment models, ie, CARPREG and ZAHARA, which consistently found that the modified WHO classification was the best available risk assessment model for estimating cardiovascular risk in pregnant women with congenital heart disease..$^{3-5}$

The modified WHO classification can be used in both congenital and acquired heart diseases. ${ }^{2}$ However, validation of the guidelines in acquired heart disease was limited. In our country, like many other developing countries, rheumatic heart disease is predominant. Before implementation of the guidelines into routine practice, validation to reaffirm the value is of importance. Therefore, we conducted this study to validate the modified WHO classification in pregnant women with both congenital and acquired heart diseases.

\section{Patients and methods}

This study was approved by the Ethics Committee of the Faculty of Medicine, Prince of Songkla University. The database of pregnant women with heart disease, who delivered at Songklanagarind Hospital between January 1995 and December 2016, was retrieved from the Statistical Unit, Department of Obstetrics and Gynecology, along with the Hospital Information System of Songklanagarind Hospital, a university hospital and a tertiary care center in southern Thailand, Faculty of Medicine, Prince of Songkla University. All medical records were reviewed. The patient consent to review their medical records was not required by the Ethics Committee of the Faculty of Medicine, Prince of Songkla University. To protect patients' confidentiality, any information which could identify patients, was not entered in the case record form.

Each patient was retrospectively classified at first visit according to the modified WHO classification of maternal cardiovascular risk by two investigators.

Baseline demographic characteristics, underlying cardiac lesion, cardiac intervention prior to pregnancy, cardiac intervention during pregnancy and postpartum period, New York Heart Association (NYHA) functional class throughout pregnancy, cardiovascular complications, echocardiography and fetal outcomes were collected. All patients were taken care of by obstetricians and cardiologists.

\section{Definition of outcomes}

Cardiovascular events were: death due to heart disease, clinical significant congestive heart failure with acute pulmonary edema requiring treatment, vascular events (eg, stroke, acute myocardial infarction or dissection), the need for urgent or invasive cardiovascular intervention during pregnancy and puerperium, symptomatic arrhythmia requiring treatment, thromboembolic events (eg, pulmonary embolism, systemic embolism, valve thrombosis or deep venous thrombosis), infective endocarditis and worsening of at least two NYHA functional classes as compared to baseline.

Offspring events were: stillbirth, early neonatal death, preterm birth (delivery $<37$ weeks' gestation), small for gestational age (birth weight $<10$ th percentile), low birth 
weight $(<2,500 \mathrm{~g})$, low Apgar scores $(<7)$ at 5 minutes and neonatal intensive care unit admission.

The Statistical Package for the Social Sciences (version 17; SPSS Inc., Chicago, IL, USA) software was used for statistical analysis. Comparison of demographic data and outcomes among the four modified WHO classes and subgroups of congenital and acquired heart diseases were analyzed using the chi-square test or Fisher's exact test and one-way ANOVA test. A $p$-value of $<0.05$ was considered statistically significant.

\section{Results}

A total of 331 cases were studied, including 157 (47.4\%) congenital heart diseases and 174 (52.6\%) acquired heart diseases (161 rheumatic heart diseases, 8 arrhythmia and 5 cardiomyopathy). The most common type of congenital heart disease was a ventricular septal defect (34.4\%), followed by an atrial septal defect $(29.3 \%)$ and then patent ductus arteriosus (13.4\%). Congenital heart disease was corrected in 50 cases (31.8\%) before pregnancy. In acquired heart disease, rheumatic heart disease was the most prevalent (92.5\%), mainly involving the mitral valve. Surgical correction was performed in 58 cases $(33.3 \%)$ before pregnancy. The percentage of referred cases was $47.4 \%$.

There were 48, 173, 32 and 78 cases in the modified WHO class I, II, III and IV, respectively. Comparison of maternal characteristics in addition to fetal outcomes among the four classes is shown in Table 1. Age and parity were not statistically different among the four classes. In the modified WHO class I, most cases were congenital heart diseases, whereas in class II-IV, acquired heart disease was more prevalent. Rates of surgical correction before pregnancy were significantly different among classes, with the lowest rate in the modified WHO class IV. Referred cases were more prevalent in class III and IV. There were significant differences among the groups in number of antenatal visits, gestational age at delivery, birth weight and means of Apgar scores at 5 minutes. Those in a higher modified WHO class had the worse fetal outcomes. Since birth weight was influenced by gestational age at delivery, we used multivariate linear regression analysis to control this factor, which found that the modified WHO class IV had significantly lower birth weight than in the class I $(\beta=-273.68$ [71.34], $p<0.001)$. Birth weight $z$-scores were also calculated, which showed a significantly decreasing trend in higher classes. Mode of delivery among groups was not statistically different. The leading indication for cesarean section was previous cesarean section (29.3\%). The leading indication for operative vaginal delivery (forceps and vacuum extraction) was to shorten second stage of labor due to maternal heart disease (90\%).

Adverse events are shown in Table 2. Maternal cardiovascular events occurred in $24.2 \%$ of cases, significantly increasing the rates with a higher modified WHO class $(p<0.001)$. Congestive heart failure was the most common complication. The overall maternal mortality rate was $3.6 \%$ $(12 / 331)$, all of whom were in the modified WHO class IV.

Table I Maternal characteristics and obstetric outcomes

\begin{tabular}{|c|c|c|c|c|c|}
\hline \multirow[t]{2}{*}{ Characteristics } & \multicolumn{4}{|c|}{ Modified WHO classification } & \multirow[t]{2}{*}{ p-value } \\
\hline & $\begin{array}{l}\text { Class I } \\
(\mathrm{N}=48)\end{array}$ & $\begin{array}{l}\text { Class II } \\
(N=\mid 73)\end{array}$ & $\begin{array}{l}\text { Class III } \\
(\mathbf{N}=32)\end{array}$ & $\begin{array}{l}\text { Class IV } \\
(\mathbf{N}=78)\end{array}$ & \\
\hline Age (years), mean (SD) & $28.7(5.8)$ & $28.1(6.5)$ & $28.4(5.7)$ & $28.8(6.8)$ & 0.86 \\
\hline Nulliparity & $56.2 \%$ & $50.3 \%$ & $37.5 \%$ & $55.1 \%$ & 0.33 \\
\hline Type of heart disease & & & & & $<0.001$ \\
\hline - Congenital & $87.5 \%$ & $46.8 \%$ & $6.2 \%$ & $41 \%$ & \\
\hline - Acquired & $12.5 \%$ & $53.2 \%$ & $93.8 \%$ & $59 \%$ & \\
\hline Surgical correction before pregnancy & $56.2 \%$ & $25.4 \%$ & $65.6 \%$ & $20.5 \%$ & $<0.001$ \\
\hline Referred cases & $33.3 \%$ & $39.9 \%$ & $50.0 \%$ & $71.8 \%$ & $<0.001$ \\
\hline Number of antenatal care visits, mean (SD) & $10.5(3.6)$ & $10.3(3.7)$ & $9.3(3.4)$ & $8.1(3.6)$ & $<0.001$ \\
\hline Gestational age at first antenatal care (weeks), mean (SD) & $12.5(6.0)$ & $13.1(6.5)$ & $12.7(6.5)$ & $14.7(6.8)$ & 0.19 \\
\hline Gestational age at delivery (weeks), mean (SD) & $37.8(2.4)$ & $37.8(1.9)$ & $36.6(2.5)$ & $36.0(2.7)$ & $<0.001$ \\
\hline Mode of delivery & & & & & 0.38 \\
\hline - Spontaneous vaginal delivery & $33.3 \%$ & $28.9 \%$ & $34.4 \%$ & $26.9 \%$ & \\
\hline - Vacuum extraction & $12.5 \%$ & $8.1 \%$ & $6.2 \%$ & $10.3 \%$ & \\
\hline - Forceps extraction & $6.2 \%$ & $22.0 \%$ & $15.6 \%$ & $12.8 \%$ & \\
\hline - Cesarean section & $47.9 \%$ & $41.0 \%$ & $43.8 \%$ & $50.0 \%$ & \\
\hline Birth weight $(g)$, mean $(S D)$ & $2,941.4(522.0)$ & $2,863.3(475.7)$ & $2,580.4(616.9)$ & $2,355.2(635.8)$ & $<0.001$ \\
\hline Birth weight z-score, mean (SD) & $0.37(0.90)$ & $0.23(0.82)$ & $-0.25(1.06)$ & $-0.64(1.09)$ & $<0.001$ \\
\hline Apgar score at 5 minutes, mean (SD) & $9.2(1.0)$ & $9.2(1.2)$ & $8.9(1.8)$ & $8.5(2.1)$ & 0.02 \\
\hline
\end{tabular}

Abbreviation: WHO, World Health Organization. 
Table 2 Adverse events according to the modified WHO classification

\begin{tabular}{|c|c|c|c|c|c|c|}
\hline & \multirow[t]{2}{*}{$\mathbf{N}(\%)$} & \multicolumn{4}{|c|}{ Modified WHO classification $\mathbf{N}=33 \mathrm{I}$} & \multirow[t]{2}{*}{$p$-value } \\
\hline & & $\begin{array}{l}\text { Class I } \\
(\mathrm{N}=48)(\%)\end{array}$ & $\begin{array}{l}\text { Class II } \\
(\mathrm{N}=173)(\%)\end{array}$ & $\begin{array}{l}\text { Class III } \\
(\mathrm{N}=32)(\%)\end{array}$ & $\begin{array}{l}\text { Class IV } \\
(\mathrm{N}=78)(\%)\end{array}$ & \\
\hline \multicolumn{7}{|l|}{ Maternal cardiovascular events } \\
\hline Congestive heart failure & $70(2 I . I)$ & 2.1 & 11.0 & 25.0 & 53.8 & $<0.001$ \\
\hline Arrhythmia & $15(4.5)$ & 2.1 & 1.2 & 6.3 & 12.8 & $<0.001$ \\
\hline Thromboembolism & $4(1.2)$ & 0 & 0 & 6.3 & 2.6 & 0.01 \\
\hline Infective endocarditis & $\mathrm{I}(0.3)$ & 0 & 0.6 & 0 & 0 & 0.82 \\
\hline Worsening of at least two NYHA functional classes & $61(18.4)$ & 2.1 & 12.1 & 18.8 & 42.3 & $<0.001$ \\
\hline Surgical intervention & $15(4.5)$ & 0 & 3.5 & 0 & 11.5 & 0.004 \\
\hline Maternal death & $12(3.6)$ & 0 & 0 & 0 & 15.4 & $<0.001$ \\
\hline Any cardiovascular event & $80(24.2)$ & 4.2 & 15 & 25 & 56.4 & $<0.001$ \\
\hline \multicolumn{7}{|l|}{ Offspring events } \\
\hline Stillbirth & $4(1.2)$ & 0 & 1.2 & 0 & 2.6 & 0.54 \\
\hline Early neonatal death & $4(1.2)$ & 2.1 & 0 & 3.1 & 2.6 & 0.20 \\
\hline Preterm birth & $74(22.4)$ & 6.2 & 15 & 37.7 & 42.3 & $<0.001$ \\
\hline Small for gestational age & $43(13.0)$ & 6.2 & 7.5 & 12.5 & 29.5 & $<0.001$ \\
\hline Low birth weight & $98(29.6)$ & 14.6 & 20.2 & 34.4 & 57.7 & $<0.001$ \\
\hline Low Apgar score $(<7)$ at 5 minutes & $14(4.2)$ & 2.1 & 2.3 & 6.2 & 9.0 & 0.08 \\
\hline Neonatal intensive care unit admission & $58(17.5)$ & 10.4 & 9.8 & 25.0 & 35.9 & $<0.001$ \\
\hline
\end{tabular}

Abbreviations: NYHA, New York Heart Association; WHO, World Health Organization.

Among 12 death cases, the causes of death were congestive heart failure (10), arrhythmia (1) and pulmonary embolism (1). Bleeding from coagulopathy occurred in three cases $(0.9 \%)$. No vascular events occurred in our study.

For offspring events, preterm birth, small for gestational age, low birth weight and neonatal intensive care unit admission were significantly different among the groups with a higher frequency in the modified WHO class III and IV (Table 2). Stillbirth and early neonatal death rates were low and not statistically different among the four classes.

Subgroup analyses of congenital and acquired heart diseases are shown in Table 3. Significantly higher cardiovascular complication rates were noted in the modified WHO class III and IV in both congenital and acquired heart diseases. Interestingly, acquired heart disease showed stronger association between the modified WHO class and maternal cardiovascular events than the congenital heart disease. In congenital heart disease, only congestive heart failure and maternal death were significantly higher in the modified WHO class III and IV (Table 3), whereas in acquired heart disease, the frequencies of congestive heart failure, arrhythmia, worsening of at least two NYHA functional classes as compared to baseline, the need for emergency surgical intervention and maternal death were significantly increased in the higher modified WHO class, especially class IV (Table 3). For adverse fetal events, both congenital and acquired heart diseases had significantly increased rates of preterm birth, small for gestational age, low birth weight and neonatal care unit admission in the modified WHO class III and IV.

\section{Discussion}

This study retrospectively simulated pregnant women with congenital and acquired heart diseases for cardiovascular risk assessment according to the modified WHO classification, so as to validate its value in the context of a tertiary care center in a developing country. The results confirmed that the modified WHO classification was useful to predict not only maternal cardiovascular risk but also offspring risk.

The European Society of Cardiology guidelines for management of cardiovascular diseases during pregnancy recommend the use of the modified WHO classification to estimate maternal risk. This risk classification integrates all known maternal cardiovascular risk factors including underlying heart disease and comorbidity. ${ }^{2}$ The patients are classified as very low risk (class I), low to moderate risk (class II), high risk (class III) and extremely high risk (class IV), in which pregnancy is contraindicated. ${ }^{2}$ In our study, acquired heart disease, mainly rheumatic heart disease, was more prevalent than the congenital one. The percentage of mothers classified in the modified WHO class IV, in which pregnancy was contraindicated, was as high as $23.5 \%$, and most of them were referred late from community or provincial hospitals. In addition, those in class III and IV had less antenatal care visits. This reflects the delay in seeking, reaching and receiving 


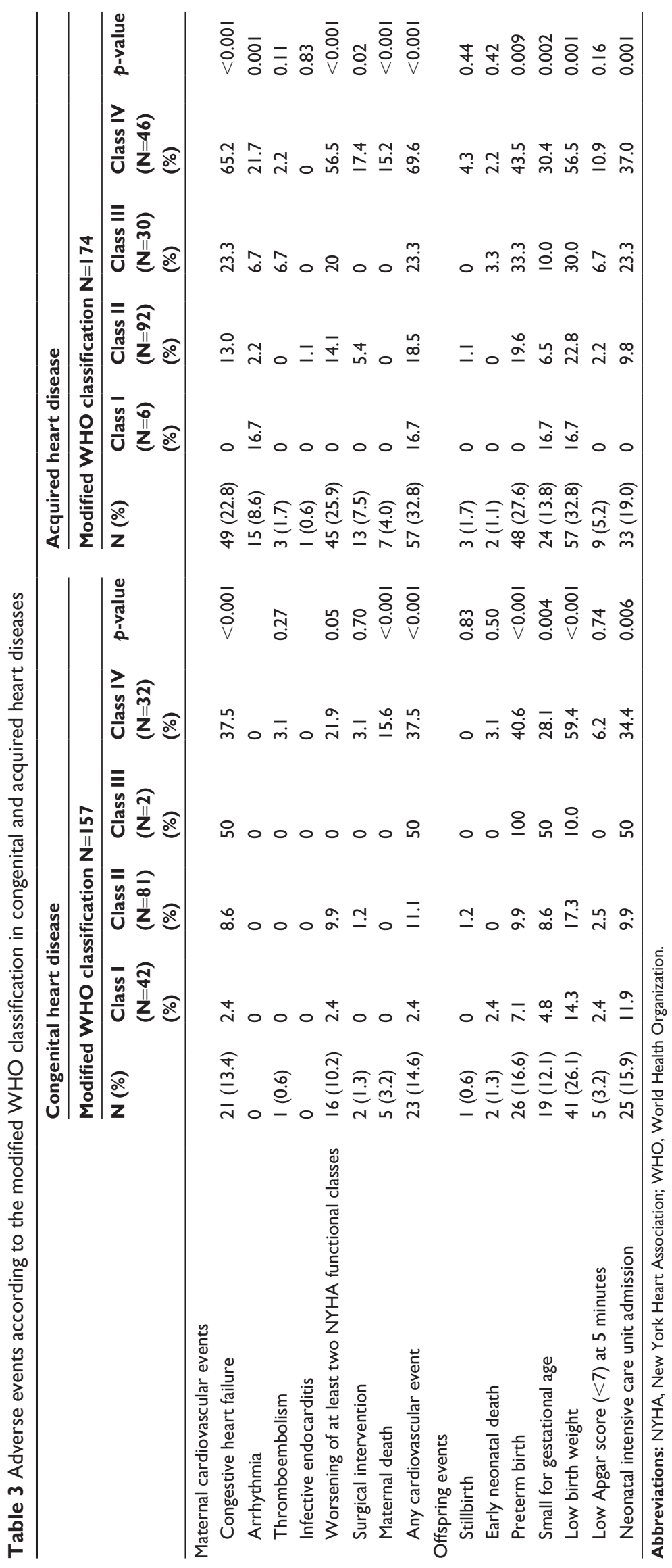


medical care in severe cases. Importantly, it decreased the opportunity to terminate a pregnancy at early gestation, especially among those classified in the modified WHO class IV. They must be evaluated for their current fitness and status before conception and counseled about anticipated complications during pregnancy with their family. Then, they should be taken care of by a multidisciplinary team in a tertiary care center at the beginning of their pregnancy.

In-depth analysis found that more women in class IV were Muslims, who faithfully believed in God and did not accept termination of pregnancy. Most of them lived in the southern border provinces and usually came to the hospitals at late gestation with severe symptoms. Another explanation of delay in receiving medical care in class IV might be a difficulty in accessing the hospital.

The overall maternal cardiovascular event rate in our study was high compared to some previous studies. ${ }^{3,6-10}$ However, it was lower than that of the study from India. ${ }^{11}$ This may have been due to the differences in clinical characteristics, such as types and severity of heart disease, maternal cardiac function, surgical correction before pregnancy, medical care received, socioeconomic status and the definition of cardiovascular events. In our study, approximately one-third of cases were in the modified WHO class III and IV. The frequency of cardiovascular events was significantly increased with the higher modified WHO class from $4.2 \%$ in class I to $56.4 \%$ in class IV. Our study had an adequate number of patients in each class to demonstrate an increasing trend of maternal cardiovascular events in all of the modified WHO classes. A recent study from Italy also found high complication rates in higher classes, 5\% in class I and II and 31\% in class III and IV. ${ }^{8}$ Detailed analysis of cardiovascular events found that congestive heart failure was the most common complication. This is similar to previous studies. ${ }^{6,7}$ The frequencies of congestive heart failure and deterioration of at least two NYHA functional classes as compared to baseline were progressively increasing from the modified WHO class I to IV.

In Thailand, although the universal health coverage system covers all medical expenditures in need by a referral system, we found that many cases were referred from community hospitals at late gestation. Several cases were in critical condition, which required emergency surgical intervention. In addition, only $20 \%$ of mothers in the modified WHO class IV had surgical correction before pregnancy. As a consequence, the maternal mortality was quite high as compared to those in the previous studies from developed countries. ${ }^{8,9}$ However, there was not much variance from that of developing countries. ${ }^{12,13}$
Subgroup analyses of congenital and acquired heart diseases have shown that pregnant women classified in the modified WHO class III and IV had higher maternal cardiovascular events in both types. Interestingly, acquired heart disease had a very high maternal cardiovascular event rate, especially in class IV (nearly 70\%), which strongly reaffirmed the value of this classification. Pregnancy must be contraindicated in the modified WHO class IV. Women with acquired heart disease seemed to have worse outcome than those with the congenital one. In-depth analysis found that women with acquired heart disease were older and more likely to be in class III and IV.

Regarding fetal outcomes, mean birth weight, gestational age at delivery and Apgar scores were significantly lower in the higher, modified WHO classes. The rates of preterm delivery, low birth weight, small for gestational age as well as admission to the neonatal intensive care unit were significantly increased in the higher classes, especially class III and IV. Subgroup analyses of congenital and acquired heart diseases also found similar results, except low Apgar score $(<7)$ at 5 minutes, which did not reach statistically significant differences. It was likely due to an inadequate sample size in subgroup analysis. Conflicting results in previous studies have been reported. One study from Italy reported lower mean birth weight, higher stillbirth rate and higher neonatal intensive care unit admission in the higher classes (III and IV) as compared to those of the lower classes (I and II). ${ }^{8}$ This is supposedly to be directly resulting from poor maternal cardiac function and severe maternal complications, which led to fetal jeopardy and also a reduction in placental supply for nutrients and oxygenation. In our study, stillbirth rate was low, so it could not show the statistical differences among the four classes. The ROPAC investigators also found a strong correlation between the modified WHO class and offspring outcome, especially preterm birth and birth weight, ${ }^{14}$ whereas the ZAHARA-II investigators could not confirm those findings. ${ }^{3}$ The possible explanation might be that the modified WHO classification was not designed to assess offspring events in mothers with congenital heart disease; therefore, known risk factors for offspring events were not taken into account. ${ }^{3}$

The strength of our study was that we had a large number of mothers with heart disease, so that it could demonstrate an increasing trend in adverse cardiovascular event rates in the modified WHO classification, from classes I to IV. In addition, it consisted of both congenital and acquired heart diseases in the context of developing country, where rheumatic heart disease was more prevalent. The results 
reaffirmed the value of the modified WHO classification not only in obtaining maternal cardiovascular risk but also in predicting adverse fetal outcomes.

The limitation of the study was that it was retrospective, and some data may have been missed. However, our data were collected in an electronic-based hospital information system of the medical school, which is concerned about the quality of the medical records and does have a quality assurance system.

We recommend that implementation of the guidelines to estimate maternal cardiovascular risk according to the modified WHO classification should be done at all levels of our health care systems, from primary to tertiary care centers. Early referral of high-risk pregnant women with heart disease to be evaluated and managed at a tertiary care center by a multidisciplinary team is an important key to improving pregnancy outcome and decreasing maternal mortality.

In conclusion, the modified WHO classification is useful not only for obtaining a cardiovascular risk assessment in pregnant women but also for predicting adverse fetal outcomes. It must, therefore, be implemented into routine care service, ideally before conception, or at the latest during the early stages of pregnancy, at all levels within the health care systems.

\section{Acknowledgments}

This study was supported by a grant from the Faculty of Medicine, Prince of Songkla University. The abstract of this paper was presented at the 25th Asian \& Oceanic Congress of Obstetrics and Gynecology 2017 as an E-poster presentation with interim findings. The poster's abstract was published in "Poster Abstracts" in the Journal of Obstetrics and Gynaecology Research, Volume 43, Issue Supplement S1, June 2017 (http://onlinelibrary.wiley.com/doi/10.1111/ jog.13388/full).

\section{Disclosure}

The authors report no conflicts of interest in this work.

\section{References}

1. Elkayam U, Goland S, Pieper PG, Silverisde CK. High-risk cardiac disease in pregnancy: part I. $J$ Am Coll Cardiol. 2016;68(4):396-410.

2. Regitz-Zagrosek V, Blomstrom Lundqvist C, Borghi C, et al. ESC guidelines on the management of cardiovascular diseases during pregnancy. Eur Heart J. 2011;32(24):3147-3197.

3. Balci A, Sollie-Szarynska KM, van der Bijl AG, et al. Prospective validation and assessment of cardiovascular and offspring risk models for pregnant women with congenital heart disease. Heart. 2014;100(17): $1373-1381$.

4. Lu CW, Shih JC, Chen SY, et al. Comparison of 3 risk estimation methods for predicting cardiac outcomes in pregnant women with congenital heart disease. Circ J. 2015;79(7):1609-1617.

5. Fu Q, Lin J. Predictive accuracy of three clinical risk assessment systems of cardiac complications among Chinese pregnant women with congenital heart disease. Int J Gynaecol Obstet. 2016;134(2):130-134.

6. Thanajiraprapa T, Phupong V. Pregnancy complications in women with heart disease. J Matern Fetal Neonatal Med. 2010;23(10):1200-1204.

7. Martins LC, Freire CM, Capuruçu CA, Nunes Mdo C, Rezende CA. Risk prediction of cardiovascular complications in pregnant women with heart disease. Arq Bras Cardiol. 2016;106(4):289-296.

8. Santacesaria S, Cataldo S, Annoni GA, Corbella P, Fiocchi S, Vignati GM Pregnancy in women with cardiovascular disease in the guidelines era: an Italian single-center experience. $J$ Cardiovasc Med. 2016; 17(10):750-755.

9. Siu SC, Sermer M, Colman JM, et al. Prospective multicenter study of pregnancy outcomes in women with heart disease. Circulation. 2001; 104(5):515-520.

10. Madazli R, Sal V, Cift T, Guralp O, Goymen A. Pregnancy outcomes in women with heart disease. Arch Gynecol Obstet. 2010;281(1):29-34.

11. Bhatla N, Lal S, Behera G, et al. Cardiac disease in pregnancy. Int $J$ Gynaecol Obstet. 2003;82(2):153-159.

12. Sawhney H, Aggarwal N, Suri V, Vasishta K, Sharma Y, Grover A. Maternal and perinatal outcome in rheumatic heart disease. Int $J$ Gynaecol Obstet. 2003;80(1):9-14.

13. Kovavisarach E, Nualplot P. Outcome of pregnancy among parturients complicated with heart disease in Rajvithi Hospital. J Med Assoc Thai. 2007;90(11):2253-2259.

14. Roos-Hesselink JW, Ruys TP, Stein JI, et al. Outcome of pregnancy in patients with structural or ischemic heart disease: results of a registry of the European Society of Cardiology. Eur Heart J. 2013;34(9): $657-665$.
International Journal of Women's Health

\section{Publish your work in this journal}

The International Journal of Women's Health is an international, peerreviewed open-access journal publishing original research, reports, editorials, reviews and commentaries on all aspects of women's healthcare including gynecology, obstetrics, and breast cancer. The manuscript management system is completely online and includes

\section{Dovepress}

a very quick and fair peer-review system, which is all easy to use. Visit http://www.dovepress.com/testimonials.php to read real quotes from published authors. 